\title{
Smell Identification Function in Children with Attention Deficit Hyperactivity Disorder
}

\author{
Ahmad Ghanizadeh ${ }^{\circledR}$, Maryam Bahrani ${ }^{1}$, Ramin Miri $^{2}$ and Ali Sahraian ${ }^{1}$ \\ ${ }^{1}$ Research Center for Psychiatry and Behavioral Sciences, Department of Psychiatry, School of Medicine, Shiraz University of Medical Sciences, \\ Shiraz, Iran \\ ${ }^{2}$ Medicinal \& Natural Products Chemistry Research Center, Shiraz University of Medical Sciences, Shiraz, Iran
}

\begin{abstract}
Objective Deficits in olfactory function are common features in neurodegenerative and neuropsychiatric disorders. Olfactory processing is related to dopamine metabolism and orbitofrontal cortex functioning, both known to be involved in the neurobiology of ADHD. Some investigations suggested alterations in olfactory processing (identification and detection threshold) in patients with ADHD. Despite increasing knowledge, controversy about this topic still exists regarding children with ADHD. This study was conducted to help elucidate some of this controversy.

Methods 50 participants $(8-15$ years, mean $=10.70 \pm 1.77)$ with ADHD were compared to 50 controls. The two groups were well matched for age, gender and Mean School Scores (MSS). We assessed odor identification and threshold through a smell test composed of two tests of identification and detection threshold. Odor detection threshold was assessed with the odorant phenyl ethyl alcohol solved in propylene glycol using a single staircase method. Odor identification was assessed with chemical essences of five common odorants.

Results The mean Sensory Identification Score for children with ADHD and the control groups were 3.76 (1.06) and 4.46 (0.76), respectively $(\mathrm{p}<0.001)$. The mean for Sensory Threshold Score for ADHD and control group was 6.4 (3.35) and 9.75 (2.16), respectively ( $<<0.001)$.

Conclusion This study replicated altered olfactory performance in ADHD. Substantial olfactory deficits across the two domains of identification and detection threshold are observed in children with ADHD. These deficits do not seem to be a result of olfactory task difficulty and are not influenced by age, gender and MSS. Further studies are required to investigate whether olfactory function can be used as a biological marker for early diagnosis, treatment and prognosis of ADHD.

Psychiatry Investig 2012;9:150-153
\end{abstract}

Key Words ADHD, Olfaction, Identification, Threshold, Smell test.

\section{INTRODUCTION}

Attention deficit hyperactivity disorder (ADHD) is a very common psychiatric disorder in children and adolescents. ADHD is associated with impairments in different domains of sensory function. ${ }^{1-6}$ Understanding sensory problems in ADHD may help us better understand the spectrum of problems included within the broad label of ADHD.

Unlike to other sensory systems, few synapses are located between the olfactory cortex and the peripheral olfactory receptors

Received: June 7, 2011 Revised: December 11, 2011

Accepted: December 16, 2011 Available online: April 30, 2012

$\triangle$ Correspondence: Ahmad Ghanizadeh, MD

Research Center for Psychiatry and Behavioral Sciences, Department of Psychiatry, School of Medicine, Shiraz University of Medical Sciences, Shiraz, Iran

Tel: +98-711-62793 19, Fax: +98-711-6279319

E-mail: ghanizad@sina.tums.ac.ir

(a) This is an Open Access article distributed under the terms of the Creative Commons Attribution Non-Commercial License (http://creativecommons.org/licenses/by$\mathrm{nc} / 3.0$ ) which permits unrestricted non-commercial use, distribution, and reproduction in any medium, provided the original work is properly cited. leading to a direct relation between brain and environment. ${ }^{2,7}$

Regarding these facts, olfactory function is increasingly being used as a marker in neurodegenerative and neuropsychiatric disorders. Olfactory dysfunction is reported in many neurologic and psychiatric disorders such as Alzheimer's disease, Parkinson's disease (PD), Schizophrenia, Autism and Obsessive compulsive disorder (OCD).$^{8-10}$ For example, odor identification, odor detection threshold and odor memory are impaired in patients with schizophrenia. ${ }^{11}$ The ability of children and adolescents with autism is less than healthy matched controls in identifying common odors. ${ }^{10}$

Olfactory function in patients with $\mathrm{ADHD}$ has been a topic of increasing interest. It is reported that processing of smells is mediated by a set of neuroanatomical and neurochemical structures that have been implicated in the pathophysiology of ADHD. ${ }^{12,13}$

Greater errors in odor identification are found in adults with $\mathrm{ADHD}$ comparing to healthy control group; although the dif- 
ference was not significant anymore when the two groups matched for IQ. ${ }^{2}$ In another study Scheckelman and colleagues reported no altered olfactory performance in adult $\mathrm{ADHD}$ patients but they found an association of cortical olfactory processes with hyperactivity and impulsivity in adult ADHD. ${ }^{14}$ Gansler reported that olfactory identification in adults with ADHD is less than the normal controls. ${ }^{15}$

There was found only two studies included children and adolescents with ADHD. Romanos et al reported significant improved odor sensitivity (threshold) in un-medicated ADHD children and adolescents compared to odor sensitivity of patients medicated with methylphenidate and healthy controls which are similar to each other. ${ }^{16}$ Others reported that olfactory identification ability of children with ADHD is significantly poorer than healthy controls. ${ }^{17}$

Moreover, a study compared odor identification between ADHD adults and a control group. There was no significant interaction between group and gender. ${ }^{2}$ Therefore, we hypoetized that there is no gender differences in olfactory processing between boys and girls with ADHD.

Overall, olfactory processing function is not typically studied in ADHD patients and the few existing researches on this topic have yielded inconsistent results. ${ }^{14,16-18}$ In general, olfactory processing in children with $\mathrm{ADHD}$, but not in adults with $\mathrm{ADHD},{ }^{19}$ seems to be impaired. Therefore, we performed the current study to assess the olfactory function of ADHD children and compare it to healthy controls. Regarding the similarity of the pathways involved in the pathophysiology of ADHD and those of olfactory processing, we hypothesized that olfaction is diminished in ADHD patients in both genders. If this comes true, future studies may wish to make exploration into whether systematic clinical assessment of olfactory function can provide valuable information for the early detection and classification of $\mathrm{ADHD}$ patients or whether this sensory domain can be used to predict course of the illness or to monitor treatment response.

\section{METHODS}

The participants were 100 children and adolescents including 50 individuals with $\mathrm{ADHD}$ and 50 children and adolescents as the control group. The two groups were match for age, gender and mean school scores (MSS). This study involved a comparison of two groups of children between the ages 8-15 (SD= 1.7) years. There were 15 girls and 35 boys in each group. All the patients did not take stimulants for at least seven hours prior to testing.

Inclusive criteria were:

1- Chronological age between 8-15 years

2- No evidence or history of recent upper respiratory tract infection, sinusitis or nasal allergies.
3- No past history of severe head trauma, hypothyroidism, neurologic disorder or gestational and birth problems.

ADHD was diagnosed using the kiddie schedule for affective disorders and schizophrenia (KSADS) according to Diagnostic and Statistical Manual of Mental Disorders, 4th. Edition (DSM-IV). ${ }^{20}$ The ADHD children and their parents were interviewed face to face. The parents of the patient participants joined the test but the healthy one's did not. Olfactory identification and threshold were assessed through a smell-test kit provided by the Center of Medicinal and Natural Products Chemistry Research affiliated to Shiraz University of Medical Sciences. The test was composed of two different tasks that were initially explained for the participants by detail. All of the children understood what they had to do. One task was to examine smell identification ability and the other one to assess smell threshold ability. The smell identification part consisted of five glass sniff-bottles, each one containing $4 \mathrm{~mL}$ of a specific odorant. Five common odorants including essences of apple, banana, lemon, orange and rose were chosen in respect to their familiarity to almost all individuals (16). The participant's task was to sniff the bottle for 3 seconds and choose the answer from a list of 4 descriptors reported by examiner. The number of correct answers was registered as the sensory identification score of the participant.

The olfactory threshold was assessed by serving a perfume grade Phenyl Ethyl Alcohol (PEA) as the odorant. The diluent used in this study was Propylene Glycol (PG) a relatively odorless and non-toxic material in which PEA is soluble (17). Detection thresholds were determined using a single staircase procedure (18). 16 triplets of bottles were prepared based on 16 different dilutions of PEA in PG in a geometric series with dilution ratio of 2/1. Each triplet was composed of three glass sniff-bottles: one of them containing $4 \mathrm{~mL}$ of a particular concentration of the odorant (PEA) dissolved in the diluent (PG), the other two bottles containing $4 \mathrm{~mL}$ of the diluents (PG) alone. The participant's task was to sniff each bottle for 3 seconds and identify the bottle that contained the odorant (or the bottle that had smell). If a correct answer was given in 2 successive trials, it would trigger the reversal of the staircase to the next lower concentration. If a wrong answer was given on any trial the next higher concentration was presented. There was a total 4 staircase reversals. The geometric mean of the last 3 reversal points was defined as the threshold estimate of the participant. The testing procedure lasted about 25 minutes. This study was approved by Shiraz university of Medical sciences. Participation was voluntary. The Univariate Analysis of Variance were used to compare the smell identification score (SIS) and smell threshold score (STS) of the two groups considering age and mean school scores covariates. Pearson correlation was performed to assess test- retest reliability. 
Table 1 .The comparison of Smell Identification Score and Smell Threshold Score between patients and controls

\begin{tabular}{lcccc}
\hline & Group & Mean (SD) & Minimum & Maximum \\
\hline Smell identification & Control & $4.46(0.76)$ & 2 & 5 \\
score & Patient & $3.76(1.06)$ & 0 & 5 \\
Smell threshold & Control & $9.75(2.16)$ & 4 & 13.6 \\
score & Patient & $6.4(3.35)$ & 0 & 13 \\
\hline
\end{tabular}

Table 2 .The comparison of Smell Identification Score \& Smell Threshold Score between genders within attention deficit hyperactivity disorder group

\begin{tabular}{lclc}
\hline & Gender & Mean (SD) & p value \\
\hline Smell identification score & Girl & $3.93(0.96)$ & 0.45 \\
& boy & $3.68(1.1)$ & \\
Smell threshold score & Girl & $6.28(3.94)$ & 0.78 \\
& Boy & $6.57(3.12)$ & \\
\hline
\end{tabular}

\section{RESULTS}

The two groups of participants did not differ in terms of age, gender and MSS. Their age ranged from 8-15 (Mean=10.8, $\mathrm{SD} \pm 1.7)$. The mean (SD) of age for the patients and control group was 10.7 (1.7) and 10.7 (1.6), respectively. The mean of age was not different between the two groups $(\mathrm{t}=0.11, \mathrm{df}=98$, $\mathrm{p}=0.9) .30 \%(\mathrm{n}=15)$ of all participants in each group were girls and $70 \%$ were boys. The mean (SD) of MSS for patients and control group was 18.1 (2.2) and $18.8(2.0)$, respectively $(\mathrm{t}=1.6$, $\mathrm{df}=98, \mathrm{p}=0.1$.

The pearson correlation between SIS results during the first and the second sessions was $(\mathrm{r}=0.55, \mathrm{p}=0.001)$. Regarding the STS the correlation results between the 2 sessions was ( $r=0.28$, $\mathrm{p}=0.10$ ).

The mean (SD) of SIS and STS are showed in Table 1. Significant differences between the two groups were evident in the two domains of olfactory function. After adjusting for age and Mean school score, both STS and SIC in ADHD group were statistically less than the control group ( $\mathrm{p}<0.001, \mathrm{p}<0.001$, respectively).

These deficits were not associated with age and Mean School Scores (MSS). None of SIS and STS was related to gender in the ADHD group (Table 2).

\section{DISCUSSION}

The findings from this clinical study show that children with ADHD have significant impairments on odor identification and odor threshold tasks compared to healthy control participants. Such findings corroborate past theoretical predictions that ADHD should be associated with olfactory dysfunction due to their neuro-anatomical and neuro-chemical similarities. Because the prefrontal lobe that is long-standing assumed to have diminished functioning in ADHD, contains primary and secondary areas for olfactory processing. These olfactory deficits are not associated with age, gender and MSS totally and are not a result of olfactory task difficulty. Another explanation for these impairments could be simply inattention of ADHD children during the olfactory task.

Prior research findings for olfactory function of children with $\mathrm{ADHD}$ have been quite inconsistent. The findings of this study lend confidence to one earlier study by Karsz et al. ${ }^{17}$ showing that patients with ADHD performed significantly worse than control participants on the olfactory identification test, however, they did not evaluate olfactory thresholds in that study and gender effects on odor identification ability could not be evaluated due to small sample size for girls. Two other studies reported no differences in olfactory function among ADHD patients compared to typical controls. ${ }^{2,14}$ One recent study suggested improved olfactory threshold in un-medicated ADHD patients compared to medicated ones and healthy controls. ${ }^{16}$ Our result is inconsistent with that study. ${ }^{16}$ We could not find any study reported this result.

Determining whether some children with ADHD have olfactory dysfunction has implications for recommending rehabilitation treatment approaches. Suggestion that olfactory dysfunction may predict other behavioral problems or psychopathy in ADHD should be explored further. Although our sample size was small to divide the group with ADHD into intact and impaired on olfactory tasks, a larger sample size may confirm that two distinct subgroups exist within the population with $\mathrm{ADHD}$ regarding olfactory function and may these subgroups are related to the traditional three subgroups mentioned for ADHD.

The current study did not support a diagnosis specific gender difference in olfactory testing. There was no differential deficit in odor identification and threshold performance between boys and girls in both patient and control groups. It is in agreement with a recent study of patients with ADHD reported that gender was not a predictor for smell detection ability in ADHD patients according to parent reports..$^{18}$ It is nevertheless contrary to results of one previous study done by Murphy et al. ${ }^{2}$ which reported that girls with ADHD performed better on olfactory identification tasks compared to boys, however, that study tested adult-age ADHD patients and current study included small sample size .

The impairment in olfactory function in ADHD patients does not seem to be a result of olfactory task difficulty, as some of participants with high MSS performed poorer on both identification and threshold tasks than some of those with low MSS. Moreover, the MSS of participants did not correlate with either SIS or STS totally; suggesting that the decline in olfactory function demonstrated in ADHD is relatively independent of the 
difficulty of the tasks. the smell test administered here prepared in accordance with the standard method described by Doty et al and Hummel et al. ${ }^{21-23}$

In sum, our results suggest olfactory deficits are present in ADHD in both domains of smell identification and smell detection threshold. These deficits are not associated with age, gender and MSS totally.

The MSS used here could better be replaced by intelligence test but it was not possible due to limitations in time and fund. The discrepant findings about olfactory function in ADHD patients that have been reported in all respective studies may be due to differences in the measures and procedures employed. Therefore, establishment of an easy administrable, worldwide affordable, and standard test of olfactory function seems warranted. In hopes that true evaluation of olfactory functioning in patients with ADHD enables us to use such assessments in early diagnosis, treatment and prognosis of the disorder. Further studies with larger sample sizes need to be conducted before a differential deficit or intact ability in these olfactory functions can be confidently declared. Future studies are required to obtain more evidence with regard to diagnostic sensitivity, specificity and validity to establish olfactory function as a biological marker of ADHD.

\section{Acknowledgments}

This study was the undergraduate thesis of Dr. Maryam Bahrani. This study was supported by a grant (No. 1943) from Shiraz University of Medical Sciences.

\section{REFERENCES}

1. Mangeot SD, Miller LJ, McIntosh DN, McGrath-Clarke J, Simon J, Hagerman RJ, et al. Sensory modulation dysfunction in children with attention-deficit-hyperactivity disorder. Dev Med Child Neurol 2001; 43:399-406.

2. Murphy KR, Barkley RA, Bush T. Executive functioning and olfactory identification in young adults with attention deficit-hyperactivity disorder. Neuropsychology 2001;15:211-220.

3. Ghanizadeh A. Tactile sensory dysfunction in children with ADHD. Behav Neurol 2008;20:107-112.

4. Ghanizadeh A. Sensory processing problems in children with ADHD, a systematic review. Psychiatry Investig 2011;8:89-94.

5. Ghanizadeh A. Can behavioral sensory processing problems guide us to a better pharmacological management of children with attention deficit hyperactivity disorder?: a case report. Psychiatry (Edgmont) 2009; 6:40-43.

6. Ghanizadeh A. Screening signs of auditory processing problem: does it distinguish attention deficit hyperactivity disorder subtypes in a clinical sample of children? Int J Pediatr Otorhinolaryngol 2009;73:81-
87.

7. Moberg PJ, Agrin R, Gur RE, Gur RC, Turetsky BI, Doty RL. Olfactory dysfunction in schizophrenia: a qualitative and quantitative review. Neuropsychopharmacology 1999;21:325-340.

8. Doty RL, Perl DP, Steele JC, Chen KM, Pierce JD Jr, Reyes P, et al. Odor identification deficit of the parkinsonism-dementia complex of Guam: equivalence to that of Alzheimer's and idiopathic Parkinson's disease. Neurology 1991;41(5 Suppl 2):77-80.

9. Boesveldt S, Verbaan D, Knol DL, Visser M, van Rooden SM, van Hilten JJ, et al. A comparative study of odor identification and odor discrimination deficits in Parkinson's disease. Mov Disord 2008;23:19841990.

10. Bennetto L, Kuschner ES, Hyman SL. Olfaction and taste processing in autism. Biol Psychiatry 2007;62:1015-1021.

11. Nguyen AD, Shenton ME, Levitt JJ. Olfactory dysfunction in schizophrenia: a review of neuroanatomy and psychophysiological measurements. Harv Rev Psychiatry 2010;18:279-292.

12. Martzke JS, Kopala LC, Good KP. Olfactory dysfunction in neuropsychiatric disorders: review and methodological considerations. Biol Psychiatry 1997;42:721-732.

13. Sabri M, Radnovich AJ, Li TQ, Kareken DA. Neural correlates of olfactory change detection. Neuroimage 2005;25:969-974.

14. Schecklmann M, Schenk E, Maisch A, Kreiker S, Jacob C, Warnke A, et al. Altered frontal and temporal brain function during olfactory stimulation in adult attention-deficit/hyperactivity disorder. Neuropsychobiology 2011;63:66-76.

15. Gansler DA, Fucetola R, Krengel M, Stetson S, Zimering R, Makary C. Are there cognitive subtypes in adult attention deficit/hyperactivity disorder? J Nerv Ment Dis 1998;186:776-781.

16. Romanos M, Renner TJ, Schecklmann M, Hummel B, Roos M, von Mering C, et al. Improved odor sensitivity in attention-deficit/hyperactivity disorder. Biol Psychiatry 2008;64:938-940.

17. Karsz FR, Vance A, Anderson VA, Brann PG, Wood SJ, Pantelis C, et al. Olfactory impairments in child attention-deficit/hyperactivity disorder. J Clin Psychiatry 2008;69:1462-1468.

18. Ghanizadeh A. The predictors of parent reported behaviors related to olfactory information processing in children with ADHD. Psychiatry Investig 2010;7:116-121.

19. Weiland R, Macht M, Ellgring H, Gross-Lesch S, Lesch KP, Pauli P. Olfactory and gustatory sensitivity in adults with attention-deficit/hyperactivity disorder. Atten Defic Hyperact Disord 2011;3:53-60.

20. Ghanizadeh A, Mohammadi MR, Yazdanshenas A. Psychometric properties of the Farsi translation of the Kiddie Schedule for Affective Disorders and Schizophrenia-Present and Lifetime Version. BMC Psychiatry 2006;6:10.

21. Doty RL, Shaman P, Dann M. Development of the University of Pennsylvania Smell Identification Test: a standardized microencapsulated test of olfactory function. Physiol Behav 1984;32:489-502.

22. Doty RL, Brugger WE, Jurs PC, Orndorff MA, Snyder PJ, Lowry LD. Intranasal trigeminal stimulation from odorous volatiles: psychometric responses from anosmic and normal humans. Physiol Behav 1978; 20:175-185.

23. Hummel T, Sekinger B, Wolf SR, Pauli E, Kobal G. 'Sniffin' sticks': olfactory performance assessed by the combined testing of odor identification, odor discrimination and olfactory threshold. Chem Senses 1997; 22:39-52. 